\title{
Single Image Dehazing Using Color Attenuation Prior
}

Qingsong Zhu ${ }^{1}$

qs.zhu@siat.ac.cn

Jiaming Mai ${ }^{1,2}$

jiamingmai@163.com

Ling Shao $^{3}$

ling.shao@ieee.org
${ }^{1}$ Shenzhen Institutes of Advanced Technology,

Chinese Academy of Sciences, Shenzhen, China

2 South China Agricultural University, Guangzhou, China

${ }^{3}$ Department of Electronic and Electrical Engineering,

The University of Sheffield, Sheffield, UK
We propose a simple but powerful prior, color attenuation prior, for haze removal from a single input hazy image. By creating a linear model for modelling the scene depth of the hazy image under this novel prior and learning the parameters of the model with a supervised learning method, the depth information can be well recovered. With the depth map of the hazy image, we can easily remove haze from a single image. Figure 1 shows an overview of the proposed dehazing method.

To describe the formation of a hazy image, the atmospheric scattering model is widely used and it can be expressed as follows:

$$
\mathbf{I}(x)=\mathbf{J}(x) \mathrm{e}^{-\beta d(x)}+\mathbf{A}\left(1-\mathrm{e}^{-\beta d(x)}\right)
$$

where $\mathbf{I}$ is the hazy image, $\mathbf{J}$ is the scene radiance representing the hazefree image, $\mathbf{A}$ is the atmospheric light, $\beta$ is the scattering coefficient of the atmosphere and $d$ is the depth of scene.

By doing a lot of experiments on the hazy images, we find the statistics that the density of the haze is positively correlated with the difference between the brightness and the saturation in a single hazy image. Since the haze density increases along with the change of scene depth in general, we can make an assumption that the depth of the scene is positively correlated with the density of the haze and we have:

$$
d(x) \propto c(x) \propto v(x)-s(x)
$$

As the difference between the brightness and the saturation can approximately represent the density of the haze, we boldly assume that the relationship among the scene depth $d$, the brightness $v$ and the saturation $s$ is linear. Based on this assumption, we can create a linear model as follows:

$$
d(x)=\theta_{0}+\theta_{1} v(x)+\theta_{2} s(x)
$$

where $d$ is the scene depth, $v$ is the brightness, $s$ is the saturation, and $\left(\theta_{0}, \theta_{1}, \theta_{2}\right)$ are the unknown linear coefficients.

In order to determine the coefficients $\left(\theta_{0}, \theta_{1}, \theta_{2}\right)$ accurately, a simple and efficient supervised learning method is used. The training data are necessary in the supervised learning method. A training sample consists of a hazy image and its corresponding ground truth depth map in our case. In order to obtain the accurate depth information as far as possible, we use the dehazing results of Kopf et al. [1] to make an inverse calculation to acquire the depth maps. In [1], Kopf used the city model from Bing to acquire the depths for the New York images and a plain 30-meter digital terrain model for the Yosemite images. To seek a solution that minimizes the difference between the scene depth $d(x)$ estimated by Equation (3) and the true depth, we minimize the following squared loss function:

$$
L=\frac{1}{n|\omega|} \sum_{i=1}^{n} \sum_{i=1}^{\omega_{i}}\left(d_{r i}\left(x_{j}\right)-\left(\theta_{0}+\theta_{1} v_{i}\left(x_{j}\right)+\theta_{2} s_{i}\left(x_{j}\right)\right)\right)^{2}
$$

Here, $n$ is the number of the training samples, $\omega_{i}$ is the size of the hazy image of the ith training sample, $|\omega|$ is the total number of the pixels of all the hazy images in the training set, $d_{r i}$ is the depth map of the ith training sample, $v_{i}$ and $s_{i}$ are the brightness channel and the saturation channel of the hazy image of the ith training sample respectively. To facilitate the calculation, we first define the two matrices $\mathbf{X}$ and $\boldsymbol{\theta}$, and combine all the $d_{r i}$ into a vector $\mathbf{D}$ as follows:

$$
\mathbf{X}=\left[\begin{array}{cccc}
1 & 1 & \cdots & 1 \\
v_{1} & v_{2} & \cdots & v_{n} \\
s_{1} & s_{2} & \cdots & s_{n}
\end{array}\right]^{\mathrm{T}} \quad \boldsymbol{\theta}=\left[\begin{array}{c}
\theta_{0} \\
\theta_{1} \\
\theta_{2}
\end{array}\right] \quad \mathbf{D}=\left[\begin{array}{lll}
d_{r 1} & \ldots & d_{r n}
\end{array}\right]^{\mathrm{T}}
$$

Now we can rewrite Equation (4) in a more concise way as below:

$$
L=\frac{1}{n|\omega|}(\mathbf{D}-\mathbf{X} \boldsymbol{\theta})^{\mathrm{T}}(\mathbf{D}-\mathbf{X} \boldsymbol{\theta})
$$

The problem of estimating the linear coefficients $\left(\theta_{0}, \theta_{1}, \theta_{2}\right)$ can be converted into the problem of solving the following equation:

$$
\frac{\partial L}{\partial \boldsymbol{\theta}}=\frac{2}{n|\omega|} \mathbf{X}^{\mathrm{T}} \mathbf{X} \boldsymbol{\theta}-\frac{2}{n|\omega|} \mathbf{X}^{\mathrm{T}} \mathbf{D}=0
$$

The solution of the equation above is given by:

$$
\boldsymbol{\theta}=\left(\mathbf{X}^{\mathrm{T}} \mathbf{X}\right)^{-1} \mathbf{X}^{\mathrm{T}} \mathbf{D}
$$

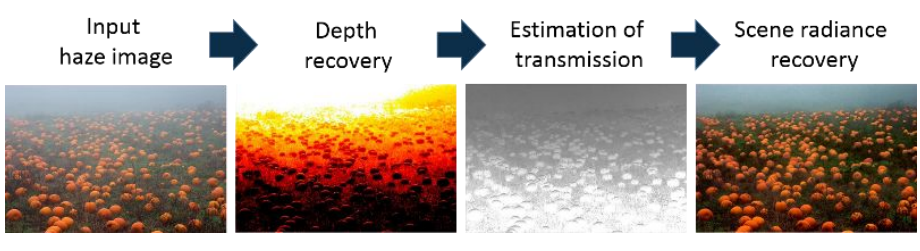

Figure 1: An overview of the proposed dehazing method.

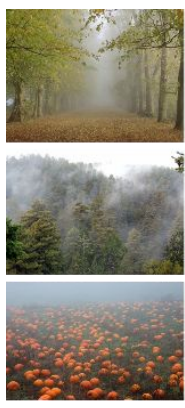

(a)

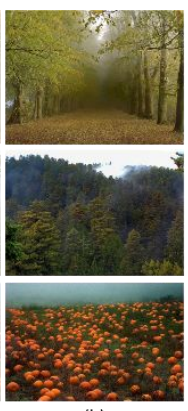

(b)

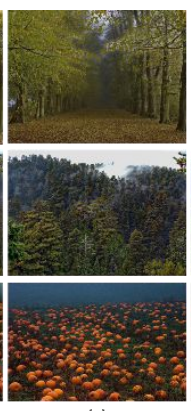

(c)

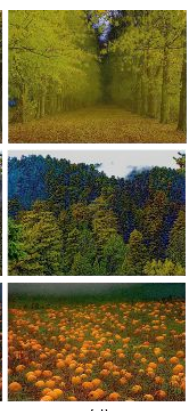

(d)

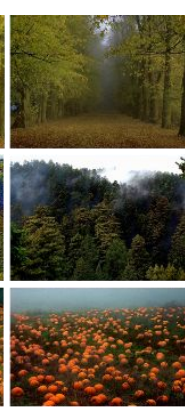

(e)
Figure 2: Comparison with other methods. (a-e) Hazy images, He et al.'s results [2], Tarel et al.'s results [3], Nishino et al.'s results [4] and ours.

We learn the linear coefficients according to Equation (8).

According to Equation (1), if $d(x) \rightarrow \infty$, then $\mathrm{e}^{-\beta d(x)} \rightarrow 0$ and $\mathbf{I}(x)=\mathbf{A}$. Based on this theory, we pick the top 0.1 percent brightest pixels in the depth map, and select the pixel with highest intensity in the corresponding hazy image I among these brightest pixels as the atmospheric light $\mathbf{A}$.

Now that the depth of the scene $d$ and the atmospheric light $\mathbf{A}$ are known, we can recover the scene $\mathbf{J}$ in Equation (1). For convenience, we rewrite Equation (1) as follows:

$$
\mathbf{J}(x)=\frac{\mathbf{I}(\mathbf{x})-\mathbf{A}}{e^{-\beta d(x)}}+\mathbf{A}
$$

where $\mathbf{J}$ is actually the haze-free image we want to obtain finally.

We implement the proposed method to test it on various hazy images and compare with the state-of-the-art methods. Figure 2 shows partial of the results. As can be seen, the dehazing effect of our method is outstanding. For an image of size $m \times n$, the complexity of the proposed dehazing algorithm is only $\mathrm{O}(m \times n)$. In Table 1 , we give the time consumption comparison with the state-of-the-art methods. As we can see, our approach is much faster than others and achieves the real-time requirement.

\begin{tabular}{|c|c|c|c|c|}
\hline Image size & He [2] & Tarel [3] & Nishino [4] & Ours \\
\hline \hline $600 \times 450$ & $12.2 \mathrm{~s}$ & $8.2 \mathrm{~s}$ & $104.7 \mathrm{~s}$ & $\mathbf{0 . 7} \mathrm{s}$ \\
$1024 \times 768$ & $36.9 \mathrm{~s}$ & $69.3 \mathrm{~s}$ & $317.4 \mathrm{~s}$ & $\mathbf{1 . 8} \mathrm{s}$ \\
$1536 \times 1024$ & $73.6 \mathrm{~s}$ & $218.0 \mathrm{~s}$ & $649.7 \mathrm{~s}$ & $\mathbf{3 . 0} \mathrm{s}$ \\
$1803 \times 1080$ & $90.7 \mathrm{~s}$ & $351.1 \mathrm{~s}$ & $861.4 \mathrm{~s}$ & $\mathbf{3 . 5} \mathrm{s}$ \\
\hline
\end{tabular}

Table 1: Time consumption comparison.

All of these experimental results show that the proposed approach is highly efficient and it outperforms the state-of-the-art haze removal algorithms in terms of the dehazing effect as well.

[1] J. Kopt, B. Neubert, B. Chen, M. Cohen and D. Cohen-Or. Deep photo: Model-based photograph enhancement and viewing. $A C M$ Transactions on Graphics, 27(5): 116, 2008.

[2] K. He, J. Sun and X. Tang. Guided image filtering. IEEE TPAMI, 35(6): 1397-1409, 2013.

[3] J. P. Tarel, and H. Nicolas. Fast visibility restoration from a single color or gray level image. In Proc. ICCV, 2009.

[4] K. Nishino, L. Kratz, and S. Lombardi. Bayesian defogging. International journal of computer vision, 98(3): 263-278, 2012. 